\title{
Competition between Abelian and Zeeman magnetic field effects in a two dimensional ultracold gas of fermions
}

\author{
Agnieszka Cichy \\ Institut für Theoretische Physik, Goethe-Universität, 60438 Frankfurt/Main, Germany \\ Krzysztof Cichy \\ Institut für Theoretische Physik, Goethe-Universität, 60438 Frankfurt/Main, Germany \\ NIC, DESY, Platanenallee 6, 15738 Zeuthen, Germany \\ Faculty of Physics, Adam Mickiewicz University, \\ Umultowska 85, 61-614 Poznań, Poland \\ Tomasz P. Polak \\ Faculty of Physics, Adam Mickiewicz University, \\ Umultowska 85, 61-614 Poznań, Poland
}

\begin{abstract}
The ground state of ultracold fermions in the presence of effects of orbital and Zeeman magnetic fields is analyzed. Five different states are found: unpolarized superconducting state, partially and fully polarized normal states and phase separated regions, partially or fully polarized. The system, in the presence of orbital synthetic magnetic field effects, shows non-monotonous changes of the phase boundaries when electron concentration is varied. We observe not only reentrant phenomena, but also density dependent oscillations of different areas of the phase diagram. Moreover the chemical potential shows oscillatory behavior and discontinuities with respect to changes in the number of fermions. Keywords: ultracold fermions, superfluidity, attractive Hubbard model, magnetic field, Zeeman effect, orbital effect
\end{abstract}

Email addresses: cichy@th.physik. uni-frankfurt.de (Agnieszka Cichy), kcichy@th.physik.uni-frankfurt.de (Krzysztof Cichy), tppolak@amu.edu.pl (Tomasz P. Polak) 


\section{Introduction}

Using quantum simulators with fully controllable Hamiltonian parameters, which can describe real situations in condensed matter physics, is a desire of the solid state community. Such simulators can reflect the main properties 5 of the physical system and with the ability to separate the effects that are indissoluble in the bulk materials, may become a standard technique. Many different many-body models are recently realized by exploiting ultracold atoms. The Bose- and Fermi-Hubbard (FH) Hamiltonians are realized with the help of optical lattices and therefore quantum phase transitions can be analyzed in great detail. The different quantum phases that exist in such systems can be detected using various techniques.

Recent experimental approaches are developed in order to simulate synthetic magnetic gauge fields, both Abelian and non-Abelian, especially in the context of quantum Hall effects. The Spielman's group succeeded in creating an external

15 synthetic magnetic, Abelian and non-Abelian, gauge field coupled to neutral gases 11]. Bloch et al. generated large tunable effective magnetic fields for ultracold atoms using photon-assisted tunneling in an optical superlattice [2, 3]. Also Ketterle et al. 4] created uniform magnetic fields with flux piercing the plaquette equal $\phi=\pi$. A major goal of these experiments is to achieve the quantum Hall regime with a very high effective flux density and to model materials where the strong correlations play the crucial role [5, 6]. This opens an avenue to study effects not achievable in conventional solid state physics 7, [8]. Experiments in which fermionic (or bosonic) gases are loaded into optical lattices have also been carried out $[9,10,11,12]$. Both the depth of the periodic

25 trapped potential and the geometry can be fully controlled. In this way, strongly correlated systems with different geometries of the lattice can be analyzed.

Another experimental aspect is the investigation of spin-polarized superfluidity in the context of cold atomic Fermi gases. They lay down one of the most investigated directions of studies in the range of condensed matter physics and ultracold quantum gases. Experimental groups from MIT [13, 14] and from 
the Rice University [15] investigated Fermi gases $\left({ }^{6} \mathrm{Li}\right)$ with unequal numbers of fermions with down $(\downarrow)$ and up $(\uparrow)$ spins $\left(N_{\downarrow} \neq N_{\uparrow}\right.$ - systems with population imbalance). Experiments have indicated the presence of a phase separation region in the system, between the unpolarized BCS and the polarized normal 35 state.

The possibility to create population imbalance in conventional superconductors can be realized by applying an external magnetic field, but this field is shielded by the orbital motion of electrons (the Meissner effect). However, a mixture with arbitrary population ratio can be prepared in atomic Fermi gases.

40 Hence, the influence of the Zeeman magnetic field on superfluidity can also be investigated in these systems.

In the presence of the Zeeman magnetic field $(h)$, the densities of states are different for the particles with spin down and spin up and there exists a mismatch between the Fermi surfaces. The population imbalance leads to

${ }_{45}$ states with nontrivial Cooper pairing. One example of such pairing is the socalled Fulde-Ferrell [16] and Larkin-Ovchinnikov [17] (FFLO) state. In this state the formation of Cooper pairs across the spin-split Fermi surface with non-zero total momentum $(\vec{k} \uparrow,-\vec{k}+\vec{q} \downarrow)$ takes place. However, the observation of such a state is very difficult in superconducting systems because of the strong so destructive influence of the orbital effect on superconductivity.

A different kind of pairing and phase coherence that can occur in such systems is the spatially homogeneous spin-polarized superconductivity (the socalled breached pair (BP) state). It is characterized by a gapless spectrum for the majority spin species $\underline{18}, 19$, , 20. The state of this type was originally con-

${ }_{55}$ sidered by Sarma 21], who studied the case of a superconductor in an external magnetic field within the BCS theory. All orbital effects were neglected. It was shown that self-consistent mean field solutions with gapless spectrum $(\Delta(h))$ are energetically unstable at $T=0$, in contrary to the fully gapped BCS solutions. On the other hand, a non-zero temperature can lead to the stabilization of a 60 spin-polarized state.

The Fermi-Hubbard system in an external Zeeman magnetic field has a very 
rich phase diagram, which was analyzed using mean field theory 22, 23, 24, 25, 26, 27, 28, 29, 30, 31], exact numerical studies (Quantum Monte Carlo (QMC) simulations and density-matrix renormalization group (DMRG) / ten-

${ }_{65}$ sor network states (TNS)) of the 1D attractive Hubbard model with fermion population imbalance [32, 33, 34, 35, 36]. These last results show that the FFLO state can be obtained in one-dimensional systems, which is consistent with the fact that $h_{c}^{F F L O}$ diverges as $T \rightarrow 0$ in $d=1$. The ground state consists of five regions: superconducting, normal and phase separated in two dimensions

7o (2D). The last two can be partially or fully polarized. The main goal of our present work is to investigate the ground state of the FH system in the presence of both Zeeman and orbital magnetic field. The presence of the Peierls factor introduces an additional change of the quantum mechanical phase of the fermionic atoms. This results not only in changes of the hopping amplitude, but

75 in consequence modifies the density of states. Engineering the Landau gauge provides a multi-band spectrum to the FH Hamiltonian with very interesting Dirac physics. We will show that taking into account the orbital magnetic field effects of electrons moving in $2 \mathrm{D}$ square lattice enriches the phase diagram and exhibits new physical phenomena.

so The paper is organized as follows. In Section II we introduce the model Hamiltonian and how the orbital effect changes the hopping term. We also briefly summarize the previously obtained results (without orbital effects) and describe main points of the method. Section III presents numerical results of our work. Section IV discusses prospects for experimental realization of the ${ }_{85}$ analyzed system. We summarize in Section V and an Appendix explains how orbital magnetic field effects can be included in the density of states.

\section{Model Hamiltonian and method of solution}

The Hamiltonian describing the spin-1/2 fermionic atoms confined in a twodimensional optical lattice can be written in the form:

$$
\mathcal{H}=\mathcal{H}_{0}+\mathcal{H}_{Z},
$$


90 where

$$
\mathcal{H}_{0}=U \sum_{i} n_{i \uparrow} n_{i \downarrow}+\sum_{i, \sigma} t_{i j} c_{i, \sigma}^{\dagger} c_{j, \sigma}-\mu \sum_{i, \sigma} n_{i, \sigma}
$$

is the pure Hubbard model, $U$ is the on-site attractive interaction, $t_{i j}$ describes the hopping matrix and $\mu$ is the chemical potential. The second term $\mathcal{H}_{Z}$ describes the effects of the external magnetic field (Zeeman) on the atoms:

$$
\mathcal{H}_{Z}=-h \sum_{i, \sigma}\left(c_{i \uparrow}^{\dagger} c_{i \uparrow}-c_{i \downarrow}^{\dagger} c_{i \downarrow}\right)
$$

The Zeeman effects give the upper limit for superconductivity, but in typical 95 condensed matter situations also the orbital effects can be important. The competition between the Zeeman and the orbital pair breaking mechanism is relevant for superconductors, since the orbital effect usually destroys superconductivity earlier than the Zeeman mechanism. The orbital effects are much more difficult to take into account and are introduced by the Peierls factor:

$$
t_{i j} \rightarrow t_{i j} e^{-i \frac{2 \pi}{\Phi_{0}} \int_{r_{j}}^{r_{i}} \mathbf{A} \cdot d \boldsymbol{l}}
$$

100 the unit cell, where $\Phi_{0}=h / 2 e$ is the elementary flux quantum. The resulting energy spectrum has Hofstadter butterfly fractal like shape [8]. Of special interest are the values of the artificial gauge field which correspond to fluxes (per unit cell)

$$
\Phi \equiv \frac{2 \pi}{k}
$$

105 with $k$ being integer. Since all properties of the Hamiltonian (2) are invariant under $\Phi \rightarrow-\Phi$ and also under $\Phi \rightarrow \Phi+\pi$, it is sufficient to consider $\Phi$ in the range $0<\Phi<\pi$ that can be reached experimentally.

The analysis of the Zeeman magnetic effects on the attractive Hubbard model was already performed [22, 37], therefore we summarize the results concentrating on the main points. The phase diagram critical lines are calculated by solving self-consistent equations for the particle number $n$, magnetization $M$ and the $s$-wave pairing symmetry order parameter $\Delta=-\frac{U}{N} \sum_{i}\left\langle c_{i \downarrow} c_{i \uparrow}\right\rangle=$ 

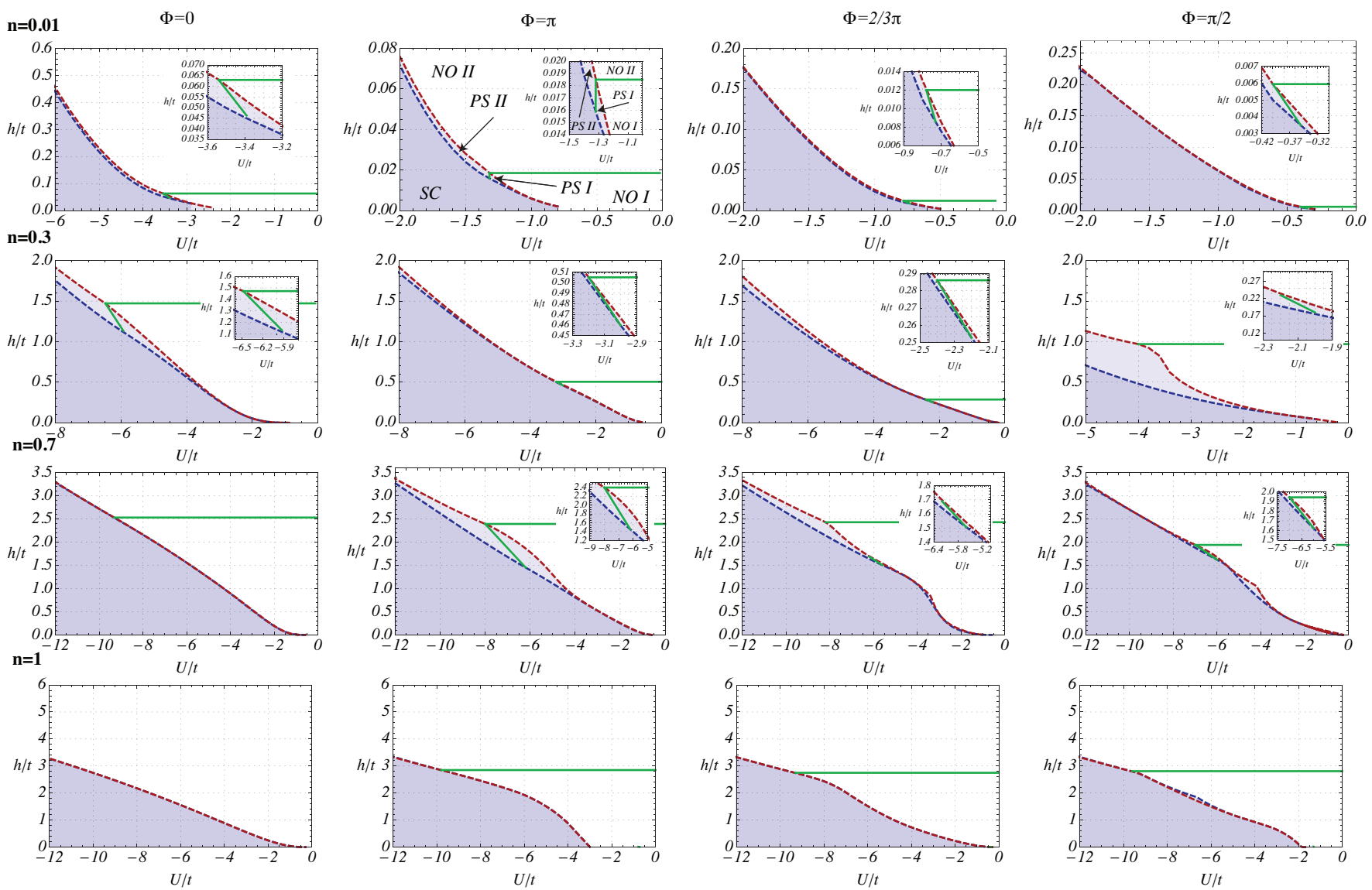

Figure 1: The ground state phase diagram for fermionic atoms confined in the two dimensional optical lattice in the absence $(\Phi=0)$ and for three values of the flux $\pi, 2 \pi / 3$ and $\pi / 2$, in the presence of the Zeeman effect. From the top, the concentration is equal $n=0.01$ (almost empty lattice) , 0.3, 0.7,1 (half filling). For clarity, we describe the phases on one plot only, but the scheme (configuration of the phases) is the same for all diagrams. SC - superconducting state, NO - normal state, PS I - partially polarized phase separation state, PS II - fully polarized phase separation state. 
$-\frac{U}{N} \sum_{\boldsymbol{k}}\left\langle c_{-\boldsymbol{k} \downarrow} c_{\boldsymbol{k} \uparrow}\right\rangle$. This system of equations, in the presence of a Zeeman magnetic field, takes the form:

$$
\Delta=-\frac{U}{N} \sum_{\boldsymbol{k}} \frac{\Delta}{2 \omega_{\boldsymbol{k}}} \frac{1}{2}\left(\tanh \frac{\beta E_{\boldsymbol{k} \uparrow}}{2}+\tanh \frac{\beta E_{\boldsymbol{k} \downarrow}}{2}\right),
$$

115 where:

$$
E_{\boldsymbol{k} \downarrow, \uparrow}= \pm \frac{U M}{2} \pm h+\omega_{\boldsymbol{k}},
$$

$\omega_{k}=\sqrt{\left.\epsilon_{k}-\bar{\mu}\right)^{2}+|\Delta|^{2}}, \bar{\mu}=\mu-\frac{U n}{2}$. We will not consider alternative phases, such as FFLO states (pairing with $\boldsymbol{q} \neq 0$ ). The particle number equation can be written as:

$$
n=1-\frac{1}{2 N} \sum_{\boldsymbol{k}} \frac{\epsilon_{\boldsymbol{k}}-\bar{\mu}}{\omega_{\boldsymbol{k}}}\left(\tanh \frac{\beta E_{\boldsymbol{k} \uparrow}}{2}+\tanh \frac{\beta E_{\boldsymbol{k} \downarrow}}{2}\right) .
$$

The equation for the magnetization is:

$$
M=\frac{1}{2 N} \sum_{\boldsymbol{k}}\left(\tanh \frac{\beta E_{\boldsymbol{k} \downarrow}}{2}-\tanh \frac{\beta E_{\boldsymbol{k} \uparrow}}{2}\right) .
$$

The first order transition lines can be determined from the condition that the grand canonical potentials of the superconducting $\left(\Omega^{S C}\right)$ and the normal states $\left(\Omega^{N O}\right)$ are equal, where the former reads:

$$
\begin{aligned}
\frac{\Omega^{S C}}{N} & =\frac{1}{4} U n(2-n)-\mu+\frac{1}{4} U M^{2}-\frac{|\Delta|^{2}}{U} \\
& -\frac{1}{\beta N} \sum_{\boldsymbol{k}} \ln \left[2 \cosh \frac{\beta\left(E_{\boldsymbol{k} \downarrow}+E_{\boldsymbol{k} \uparrow}\right)}{2}\right. \\
& \left.+2 \cosh \frac{\beta\left(E_{\boldsymbol{k} \downarrow}-E_{\boldsymbol{k} \uparrow}\right)}{2}\right]
\end{aligned}
$$

and its corresponding free energy: $F^{S C} / N=\Omega^{S C} / N+\mu n$. The grand canonical potential of the normal state is $\Omega^{S C}$ with $\Delta=0$ (entering explicitly and through $\left.E_{\boldsymbol{k} \downarrow, \uparrow}\right)$. Note that the results are obtained at a fixed chemical potential. However, they can be mapped to the case of a fixed concentration of electrons.

The details of introducing of the Peierls factor and its consequences are analyzed in Appendix A

In our numerical calculations, the sums over the first Brillouin zone have been performed with the use of the density of states, which is discussed in the Appendix (Sec. Appendix A). Consequently, the sums over the two-dimensional 
momenta $k$ are replaced by sums over energies, which considerably simplifies numerical treatment.

All phase diagrams are constructed without the Hartree term. For the system without orbital effects $(\Phi=0)$, different kinds of solutions can be obtained: the unpolarized superconducting ground state $(\mathrm{SC})$, the Pauli paramagnetic case (the normal phase NO), the region of phase separation (PS). Both the normal and phase separated areas can be partially (NO-I, PS-I, respectively) or fully polarized (NO-II, PS-II). For completeness, we show the $\Phi=0$ results in Fig. [1 together with results for $\Phi \neq 0$. We consider the case of a fixed electron concentration, which results in two critical Zeeman fields constraining the phase separation regions on the phase diagram. The particle-hole symmetry implies that the range of phase diagrams can be restricted to $n \in[0,1]$. The results for the low density limit (Fig. $n=0.01, \Phi=0$ ) have been discussed in the context of the BCS-BEC crossover in Ref. 22]. Here, we summarize the main points. From the point of view of the BCS-BEC crossover, the construction of the phase diagrams which describe the evolution of the system with increasing attraction is very important. unmagnetized $\mathrm{SC}_{0}$ phase was found in the strong attraction limit. With increasing magnetic field, PS is energetically favored i.e. even in the strong attraction limit, some unconventional, spatial homogeneous phases (like the Sarma or breached (BP-1) phase) are unstable. Superconductivity is destroyed by pair breaking in the weak coupling regime. In the strong coupling regime, the transition from the superconducting to the normal state goes in addition through phase separation ( $\mathrm{SC}_{0} \rightarrow$ PS-II $\rightarrow$ NO-II). In the dilute limit,

155 the lattice effects are smaller and the lattice model gives results similar to those of the continuum model [38].

\section{Results}

\subsection{Ground state}

In this section, we concentrate on the properties of the system when, in addition to the Zeeman field, we consider the influence of the orbital magnetic effects 
on fermionic atoms. We restrict ourselves to the cases where closed analytical formulae for the tight binding spectrum and density of states are available [39] (see also the Appendix). The orbital effects impose a Peierls factor on the electronic wave function. Choosing the Landau gauge $\boldsymbol{A}=B(0, x, 0)$ results in the synthetic magnetic field $\boldsymbol{B}$ aligned along the $z$-axis. This is a typical situation in condensed matter physics, although in experiments with bosons confined in 2D optical lattices more options are available, due to the photon assisted tunneling technique, such as uniaxially ordered fluxes or checkerboard configurations. The miscellaneous quantum phases presented in such systems can be determined by the time-of-flight images [40].

Although the behavior of the system under synthetic magnetic field is similar for all analyzed fluxes $\Phi$, some subtleties appear which are essential to our further analysis. Therefore we must distinguish between $\pi, \pi / 2$ and $2 \pi / 3$ cases. We start from the almost empty optical lattice with a small density of fermions $75=0.01$ (see Fig 1). For all flux values, the orbital effects have a profound impact on the phase diagrams. We can, as in the case where only pure Zeeman field is considered, discriminate the normal, superconducting and phase separated regions with full and partial polarization (Fig. 10. We notice that the superconducting state is more robust against the orbital effects of the magnetic fields than the phase separated phases. We postpone the justification of this property to the next section. The position of the critical lines strongly depends on the fermionic concentration $n$ and shows a non-monotonous behavior also discovered in bosonic systems [41, 39]. The phase separated areas, very small for low fermionic concentrations are enlarged and again reduced when we add ${ }_{85}$ more particles to the system. These "oscillations" are better visible for the $2 \pi / 3$ and $\pi / 2$ fluxes in comparison with the $\pi$ flux (Fig. 2 and Fig. 31). When the flux density reaches $2 \pi / 3$, the phase separated regions are more stable than in the other cases. This behavior is the consequence of the van Hove singularity that appears in the middle of the density of states in the cases where the denominator in Eq. (5) is odd, in contrast to the fluxes $\pi$ and $\pi / 2$ (see Fig. [5). The area of the phase separation is increased the most for the $\pi / 2$ flux. 

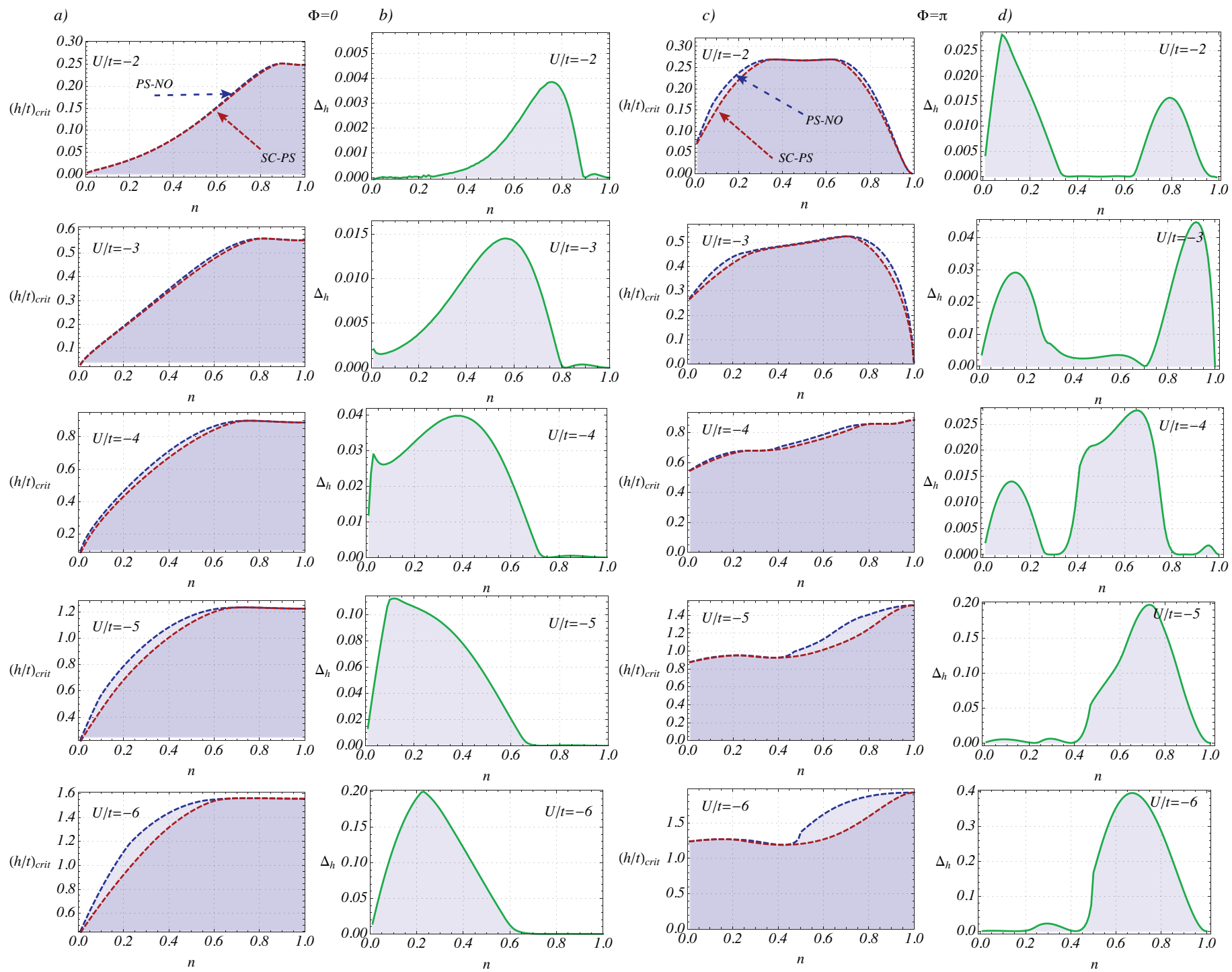

Figure 2: 1st and 3rd columns - the Zeeman critical field $(h / t)_{\text {crit }}$ vs. fermionic concentration $n$ for different values of the on-site interaction without synthetic magnetic field and for the flux $\Phi=\pi$. 2nd and 4th columns - the difference $\Delta_{h}=(h / t)_{\text {crit }}^{P S-N O}-(h / t)_{\text {crit }}^{S C-P S}$ between critical values of the Zeeman field for phase separated - normal and superconducting - phase separated boundaries $(U / t$ fixed). 


\subsection{Reentrant phenomena}

Now, we turn our analysis to the main point of our work and we focus on reentrant phenomena in the ultracold fermionic gases when the orbital effects are taken into account. Firstly, we fix the ratio $U / t$ and take the critical Zeeman field values from the boundary between the SC and the PS phases and between the PS and the NO phases. Next, we vary the fermionic concentration (Fig. [2] and 3). We observe the increase of the critical value of the Zeeman field $(h / t)_{\text {crit }}$ as the population of electrons is growing. When only Zeeman effects are considered, the critical field $(h / t)_{\text {crit }}$ is monotonously concentration dependent. However, the situation is dramatically changed when the synthetic magnetic field is applied to the system. For small attractive interaction strength, the behavior of the ultracold fermions is very different than in the absence of the flux. When we increase the interaction between fermions for $\Phi=0$ and $\pi$, the $(h / t)_{\text {crit }}$ starts to oscillate and for $U / t>4$ the system exhibits reentrant behavior which is even more pronounced for the lower magnetic fields. Therefore, we argue that when the fluctuations of the concentration are large, the system can spontaneously change the phases and oscillate between them. The stronger the attractive interaction is, the more pronounced the oscillations are. Moreover, there is also an asymmetry in the behavior of the phase boundaries SC-PS and PS-NO - although qualitatively similar, quantitatively they are rather different. The area of the phase separated region of the phase diagram is enlarging with the increase of the on-site interaction between fermions. We do not analyze finite temperatures phase diagrams, however similar behavior (critical temperature versus flux oscillations) was found in the limit of strong magnetic fields [42].

The next step to confirm our findings and to prove that the oscillations take place is to investigate the chemical potential $\mu / U$ dependence on the fermion concentration at the boundaries SC-PS and PS-NO, for fixed Zeeman field and on-site interaction energy. Although monotonically growing, we can observe some interesting behavior of the chemical potential $\mu / U$. For the $\pi$ flux and $U / t>4$ the system starts to show the oscillating behavior. Analyzing the data from numerical calculations, we notice that the chemical potential has 
lower value for the phase separated - normal state case. Still, for some electron concentrations, the energy needed to add or subtract particles from the system is changing from one phase to the other, favoring the superconducting - phase separated scenario and finally one reenters the initial state, although with larger ratio of $\mu / U$. The number of changes between different favorable scenarios does not depend on the flux density and always equals two. We also notice that the discontinuities appear for some special values of the fermionic concentration. It can be seen in Fig. [4 that the jumps in the chemical potential for large $U / t$ can be described by the simple formula giving rational values:

$$
n=\frac{1}{2 \pi} \Phi,
$$

where the position of the jump depends on the flux that pierces the unit cell. However, for weakly interacting system, we observe deviations from that expression and notice that the discontinuities appear for flux $\Phi=2 \pi / 3$ when the ${ }_{235}$ concentration reaches $n=2 / 3$, which is smeared out with growing interactions. On the other hand, for the flux $\Phi=\pi / 2$, we observe the jumps of $\mu / U$ for $n=1 / 4,1 / 2$ and $3 / 4$, which suggests that the formula:

$$
n=\frac{l}{2 \pi} \Phi
$$

with $l$ being integer, will describe all possible positions of the chemical potential discontinuities. The above phenomena represent the fact that the number of states is equally distributed among Hofstadter sub-bands (Fig. 廌). The results are also consistent with theories developed in the tight binding regime for noninteracting systems [7], where the population of each band is simply divided by the denominator in Eq. (5). Using the above, we can easily discriminate which band is filled by the electrons.

${ }_{245}$ We also notice the behavior mentioned in the previous section, namely the superconducting state is most robust against the effects of the orbital magnetic fields (Fig. 4). The boundary SC-PS is an almost monotonous function of the concentration, whereas the PS-NO boundary shows oscillatory behavior. In the superconducting phase, the strong correlations prevent the system from being 
250 penetrated by the fluxes up to some limit. Therefore, we argue that what causes the oscillations is the presence of the normal phase.

\section{Experimental realizations}

The reentrant behavior and oscillations we discuss strongly depend on the position of the Fermi level. The characteristic step in the momentum distribution for an ideal gas can be lost in the experiments with the nonuniform density. The comparison between experimental data and calculated quantities can be made, however, only in terms of averages over the density distribution. Currently, the cold atom experiments occur in an inhomogeneous harmonic trap. Usually the uniform-case results are converted, using the local density approximation, to results for a trapped gas. This can be overcome, however, by exploiting another technique used to obtain a quasi uniform three-dimensional condensate, to avoid non-uniformity usually produced by harmonic traps. The optical-box trap is formed by the three beams resulting in a box repulsive potential in which the atoms are confined. This box trap is essential in condensed

265 matter theories for homogeneous systems. The above method can be used to cut off by the dark box as many layers as needed [43]. In result, we can simulate homogeneous systems which are closer to the relevant Hubbard Hamiltonians. We notice that although highly controllable environment of the optical setups allows for modeling quite broad spectrum of the Hamiltonians, however, some effects cannot be directly observable [44] when one includes a harmonic trap, which results in an inhomogeneous system.

The phases we discuss can be checked using in situ absorption images already used to observe pairing in a gas of fermionic atoms with unequal numbers of two components. It has been shown that the gas separates into two phases: 275 superfluid core and surrounding area of the unpaired fermions [15]. Therefore, this experimental technique could serve as a good probe for the phase boundaries of the calculated phase diagrams.

In the optical lattices (periodic potential), the Hubbard limit has been 


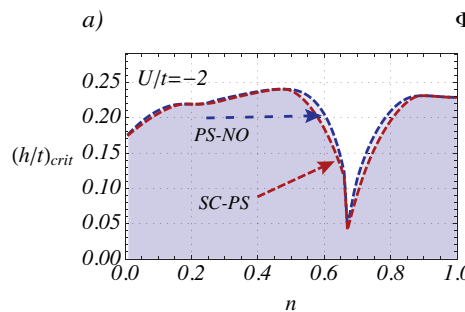

$\Phi=2 / 3 \pi \quad b)$
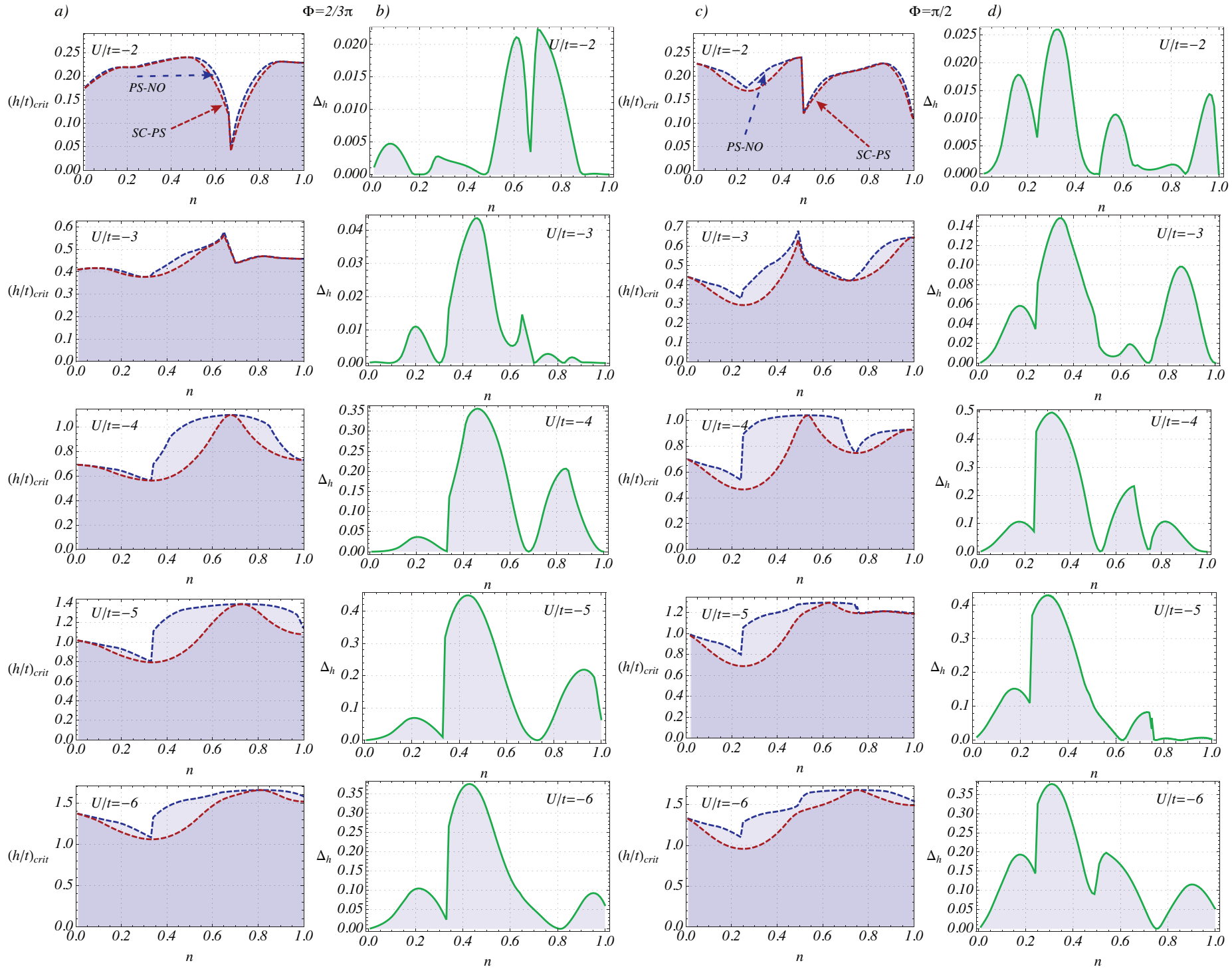

Figure 3: The same as in Fig. 2 but for the fluxes $\Phi=2 \pi / 3$ and $\pi / 2$. 

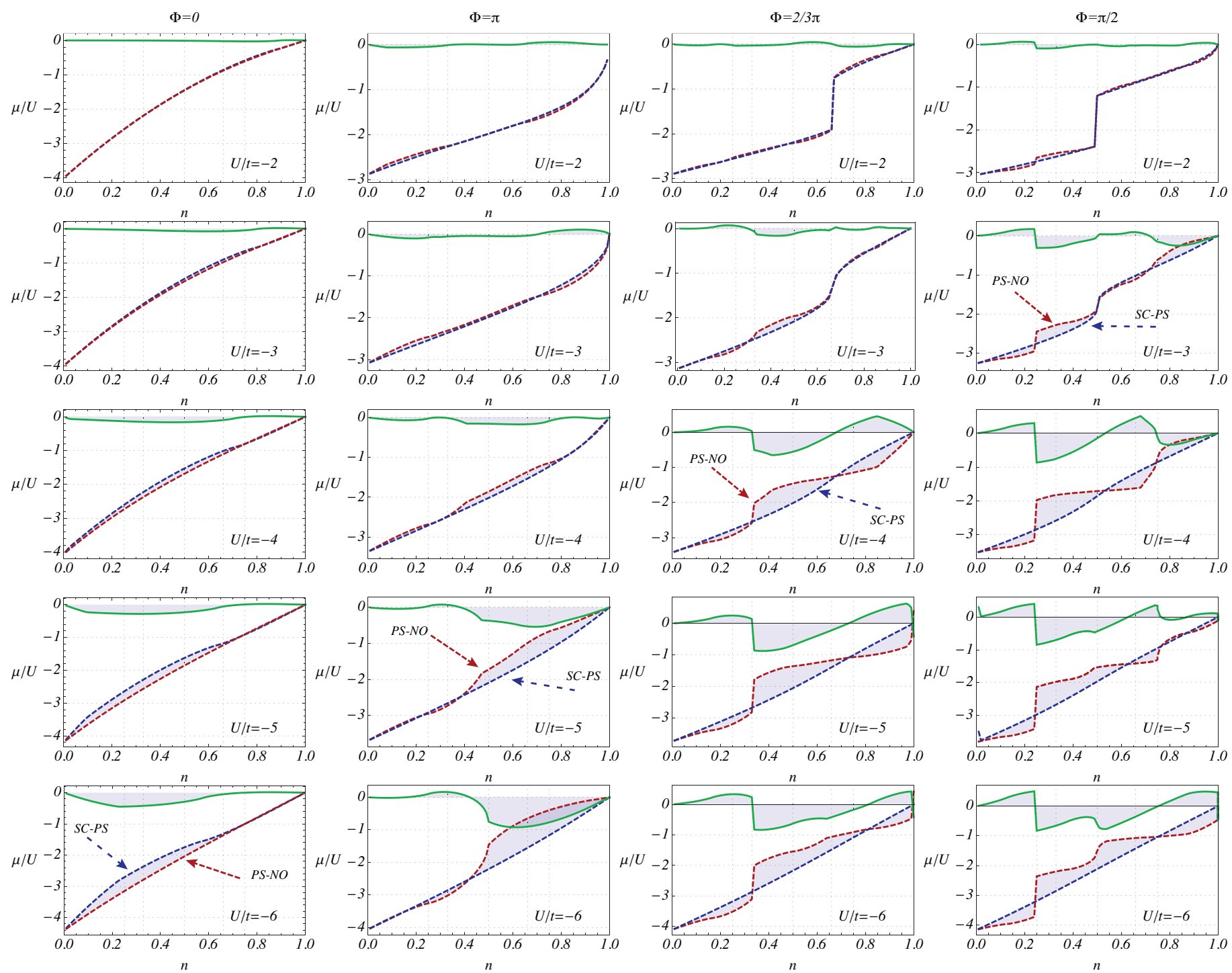

Figure 4: The dependence of the chemical potential $\mu / U$ on the fermionic concentration $n$ for different on-site interaction strengths $U / t$ and flux densities $\Phi$. Dashed lines describe the phase boundaries between phase separated - normal and superconducting - phase separated regions. The uppermost curve determines the difference between chemical potential on the phase boundaries of PS-NO and SC-PS. 

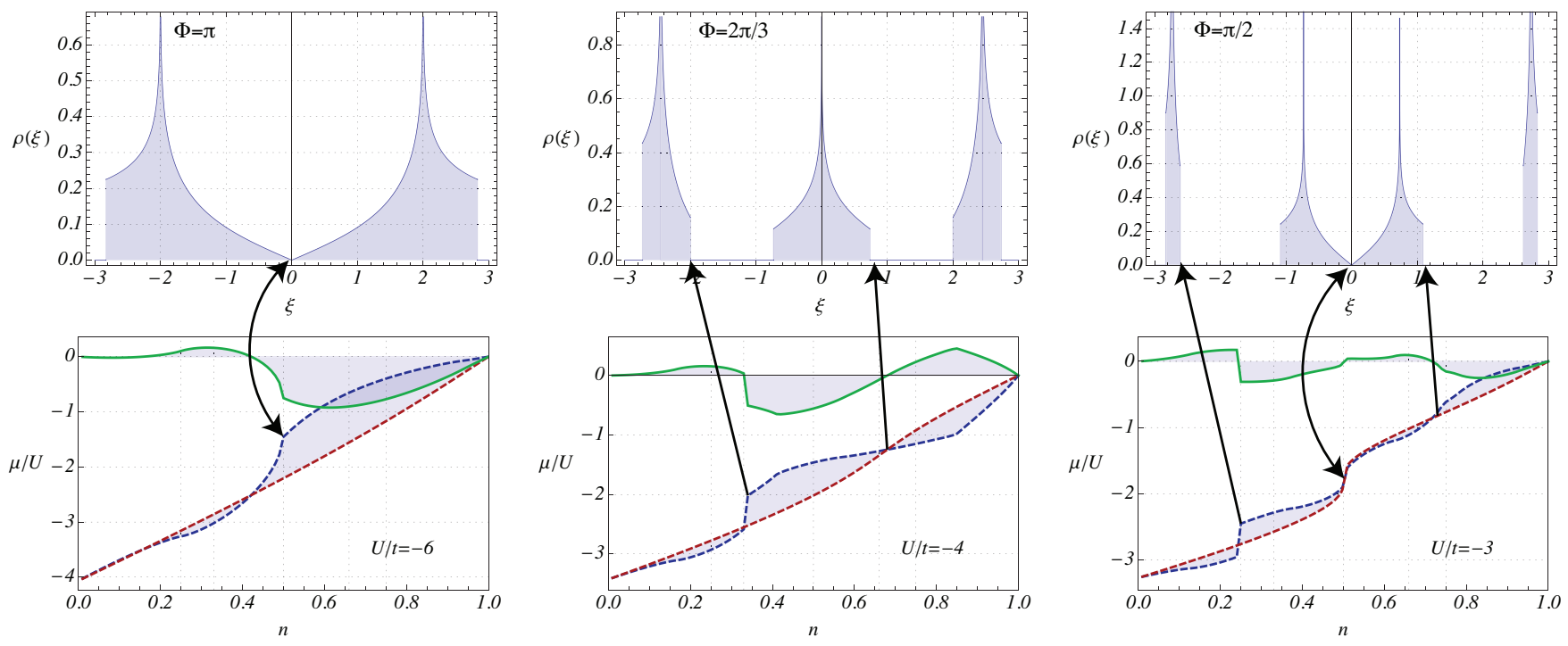

Figure 5: The equally distributed number of available states among Hofstadter sub-bands (upper row) results in the discontinuities at the special points $n=l \Phi / 2 \pi$ with $l$ being a positive integer in the chemical potential vs. concentration diagrams (lower row).

achieved only when using ${ }^{6} \mathrm{Li}$ atoms 13 . The experiments have been carried out in a superfluid regime of strong attractive interactions and long range phase coherence has been shown by observing sharp interference peaks during ballistic expansion. However, the analysis of the experiments cannot be done using a single band description [45]. The progress made in experimental techniques will allow for an experimental realization of the Hubbard Hamiltonian within the desired range of parameters in the near future.

\section{Summary}

We have analyzed the ground state of a system of ultracold fermionic atoms confined in a two-dimensional optical square lattice in the presence of two different kinds of an external magnetic field: the Zeeman field and the orbital field.

We found five phases on the phase diagram: unpolarized superconducting state, partially and fully polarized normal states and phase separated regions also partially and fully polarized. We observed oscillations of the area of the phase 
separated region with the changes of the electron concentration, which strongly depend on the flux applied to the system. As a result, reentrant behavior is found. This behavior varies with the flux density and the oscillations we notice depend on the structure of van Hove singularities and on the multi-band structure of the quasiparticle spectrum of the system. We proposed a simple formula to discriminate the position of the chemical potential jumps, which identifies which sub-band of the Hofstadter spectrum is filled. We also found that the normal state as the less correlated one is the most susceptible to the effects of the orbital magnetic fields.

\section{Acknowledgments}

T.P.P. thanks M. Mierzejewski for fruitful discussions. We also thank W. Hofstetter for carefully reading the manuscript and valuable comments.

\section{Appendix A. Orbital effects in the density of states}

We rewrite the Hamiltonian (2) in the non-interacting limit $U=0$ :

$$
\begin{aligned}
\mathcal{H}_{\mathcal{O}} & =\sum_{i, \sigma} t_{i j} e^{-i \frac{2 \pi}{\Phi_{0}} \int_{r_{j}}^{r_{i}} \mathbf{A} \cdot d l} c_{i, \sigma}^{+} c_{j, \sigma} \\
& -\mu \sum_{i, \sigma} n_{i, \sigma}-h \sum_{i, \sigma}\left(c_{i \uparrow}^{+} c_{i \uparrow}-c_{i \downarrow}^{+} c_{i \downarrow}\right) .
\end{aligned}
$$

introducing the Peierls factor that describes the orbital magnetic field effect. Assuming the Landau gauge $\boldsymbol{A}=B(0, x, 0)$, we write Eq. (A.1) in momentum space

$$
\begin{aligned}
\mathcal{H}_{\mathcal{O}} & =-t \sum_{\boldsymbol{k}, \sigma}\left[2 c_{\boldsymbol{k} \sigma}^{+} c_{\boldsymbol{k} \sigma} \cos k_{x} a+e^{-i k_{y} a} c_{\boldsymbol{k}-\boldsymbol{k}^{\prime}, \sigma}^{+} c_{\boldsymbol{k}, \sigma}\right. \\
& \left.+e^{i k_{y} a} c_{\boldsymbol{k}+\boldsymbol{k}^{\prime}, \sigma}^{+} c_{\boldsymbol{k}, \sigma}+\mu n_{\boldsymbol{k}, \sigma}\right] \\
& -h \sum_{\boldsymbol{k}}\left(c_{\boldsymbol{k}, \uparrow}^{+} c_{\boldsymbol{k}, \uparrow}-c_{\boldsymbol{k}, \downarrow}^{+} c_{\boldsymbol{k}, \downarrow}\right)
\end{aligned}
$$


310 where $\boldsymbol{k}^{\prime}=\left(2 \pi \frac{p}{q}, 0\right)$. When $p$ and $q$ are co-prime integers, we can define a magnetic lattice with an enlarged unit cell penetrated by $p$ flux quanta. In such a system, the eigenfunctions and magnetic translation operators can be found simultaneously. It can be seen that the Brillouin zone is reduced along the $y$ axis to $\left|k_{y}\right| \leq \pi / q a$, whereas nothing is changed along $x$ as a consequence of the gauge 315 chosen. In order to rewrite the Hamiltonian as a sum over a reduced (magnetic) Brillouin zone (MBZ), we introduce a multicomponent Nambu spinor:

$$
C_{\boldsymbol{k}}^{+}=\left(c_{\boldsymbol{k}, \uparrow}^{+}, c_{-\boldsymbol{k}-\boldsymbol{k}^{\prime}, \uparrow}^{+}, \ldots, c_{\boldsymbol{k}+2 \boldsymbol{k}^{\prime}, \downarrow}, c_{-\boldsymbol{k}+(q-1) \boldsymbol{k}^{\prime}, \downarrow}\right) .
$$

Now the Hamiltonian can be written in the form:

$$
\mathcal{H}_{\mathcal{O}}=\sum_{\boldsymbol{k} \in M B Z} C_{\boldsymbol{k}}^{+} \mathcal{H}_{\boldsymbol{k}} C_{\boldsymbol{k}}
$$

with

$$
\mathcal{H}_{\boldsymbol{k}}=\left[\begin{array}{cc}
\mathcal{T}_{\boldsymbol{k}, \uparrow} & 0 \\
0 & \mathcal{T}_{\boldsymbol{k}, \downarrow}
\end{array}\right] .
$$

The above matrix, for the assumed $s$-wave pairing symmetry of the order parameter $\Delta$, is diagonal. It resembles the form derived by Hofstadter [8] and describes the non-interacting fermions under a strong magnetic field. Now, we must diagonalize Eq. A.5 where:

$$
\mathcal{T}_{\boldsymbol{k}, \sigma}=-t\left(\begin{array}{ccccc}
M_{1, \sigma} & -e^{i k_{x} a} & \cdots & 0 & -e^{-i k_{x} a} \\
-e^{-i k_{x} a} & M_{2, \sigma} & \ddots & \ddots & 0 \\
0 & -e^{-i k_{x} a} & \ddots & \ddots & \vdots \\
0 & \ddots & -e^{i k_{x} a} & M_{q-1, \sigma} & -e^{i k_{x} a} \\
-e^{i k_{x} a} & 0 & 0 & -e^{-i k_{x} a} & M_{q, \sigma}
\end{array}\right),
$$

where:

$$
M_{n}=-2 \cos \left[k_{y} a+2 \pi p(n-1) / q\right]-\mu+\sigma h
$$

obtaining eigenenergies, where $\sigma= \pm 1$ represents spin up and down, respec-

325 tively. On the other hand, we can also calculate the lattice Green's functions (density of states):

$$
\rho(\xi)=\frac{1}{N} \sum_{k} \sum_{n} \delta\left(\xi-\epsilon_{\boldsymbol{k}}^{n}\right),
$$


where $\epsilon_{\boldsymbol{k}}$ are quasiparticle energies and $n$ is the number of the bands, to avoid the need for numerically solving Eq. A.5 and provide a more detailed solution. This method can be used by exploiting the already available analytical solutions for the cases $\Phi=\pi, 2 / 3 \pi$ and $\pi / 2$ [46, 39]. Therefore, in the set of self-consistent equations for the s-wave pairing symmetry case in the presence of a Zeeman magnetic field, we apply the substitution:

$$
\frac{1}{N} \sum_{\boldsymbol{k}} f\left(\epsilon_{\boldsymbol{k}}\right) \rightarrow \frac{1}{N} \int d \xi \delta\left(\xi-\epsilon_{\boldsymbol{k}}\right) f\left(\epsilon_{\boldsymbol{k}}\right)=\int d \xi \rho(\xi) f(\xi),
$$

which results in less involved numerical calculations, providing, however, the Hofstadter butterfly spectrum into the system. We must emphasize that the above can also be extended to a more general, site dependent pairing symmetry of the order parameter $\Delta_{i j}$. From that point of view, Eq. A.5 becomes nondiagonal:

$$
\mathcal{H}_{k}=\left[\begin{array}{cc}
\mathcal{T}_{k, \uparrow} & \Delta_{k} \\
\Delta_{k}^{*} & \mathcal{T}_{k, \downarrow}
\end{array}\right]
$$

and has to be solved self-consistently with Eq. [6]. However, the density of states method can still be used.

\section{References}

[1] Y. J. Lin, R. L. Compton, K. Jiménez-García, J. V. Porto, I. B. Spielman, Synthetic magnetic fields for ultracold neutral atoms, Nature 462 (7273) (2009) 628.

[2] M. Aidelsburger, M. Atala, S. Nascimbène, S. Trotzky, Y. A. Chen, I. Bloch, Experimental Realization of Strong Effective Magnetic Fields in an Optical Lattice, Phys. Rev. Lett. 107 (25) (2011) 255301.

[3] M. Aidelsburger, M. Atala, S. Nascimbène, S. Trotzky, Y.-A. Chen, I. Bloch, Experimental realization of strong effective magnetic fields in optical superlattice potentials, arXivarXiv:1212.2911v1 
350 [4] M. Hirokazuu, G. A. Siviloglou, C. J. Kennedy, W. C. Burton, W. Ketterle, Realizing the Harper Hamiltonian with Laser-Assisted Tunneling in Optical Lattices, Phys. Rev. Lett. 111 (1) (2013) 185302.

[5] K. Osterloh, M. Baig, L. Santos, P. Zoller, M. Lewenstein, Cold Atoms in Non-Abelian Gauge Potentials: From the Hofstadter "Moth" to Lattice Gauge Theory, Phys. Rev. Lett. 95 (1) (2005) 10403.

[6] P. Hauke, O. Tieleman, A. Celi, C. Ölschläger, J. Simonet, J. Struck, M. Weinberg, P. Windpassinger, K. Sengstock, M. Lewenstein, A. Eckardt, Non-Abelian Gauge Fields and Topological Insulators in Shaken Optical Lattices, Phys. Rev. Lett. 109 (14) (2012) 145301.

[7] Y. Hasegawa, P. Lederer, T. M. Rice, P. B. Wiegmann, Theory of electronic diamagnetism in two-dimensional lattices, Phys. Rev. Lett. 63 (8) (1989) 907.

[8] D. R. Hofstadter, Energy levels and wave functions of Bloch electrons in rational and irrational magnetic fields, Phys. Rev. B B14 (6) (1976) 22392249 .

[9] C. Chin, A. Altmeyer, S. Riedl, S. Jochim, J. H. Denschlag, R. Grimm, Observation of the Pairing Gap in a Strongly Interacting Fermi Gas, Science 305 (cond-mat/0405632) (2004) 1128.

[10] M. Köhl, H. Moritz, T. Stöferle, K. Günter, T. Esslinger, Fermionic Atoms in a Three Dimensional Optical Lattice: Observing Fermi Surfaces, Dynamics, and Interactions, Phys. Rev. Lett. 94 (8) (2005) 080403.

[11] M. Greiner, O. Mandel, T. Esslinger, T. W. Hänsch, I. Bloch, Quantum phase transition from a superfluid to a Mott insulator in a gas of ultracold atoms, Nature 415 (6867) (2002) 39-44.

[12] R. Jördens, N. Strohmaier, K. Günter, H. Moritz, T. Esslinger, A Mott insulator of fermionic atoms in an optical lattice, Nature 455 (7210) (2008) 204. 
[13] J. K. Chin, D. E. Miller, Y. Liu, C. Stan, W. Setiawan, C. Sanner, K. Xu, W. Ketterle, Evidence for superfluidity of ultracold fermions in an optical lattice, Nature 443 (7114) (2006) 961.

[14] C. H. Schunck, Y. Shin, A. Schirotzek, M. W. Zwierlein, W. Ketterle, Pairing without superfluidity: The ground state of an imbalanced Fermi mixture, Science 316 (5826) (2007) 867-870.

[15] G. B. Partridge, W. Li, R. I. Kamar, Y.-a. Liao, R. G. Hulet, Pairing and phase separation in a polarized Fermi gas, Science 311 (5760) (2006) 503-505.

[16] P. Fulde, R. A. Ferrell, Superconductivity in a Strong Spin-Exchange Field, Phys. Rev. 135 (1964) A550-A563.

[17] A. I. Larkin, Y. N. Ovchinnikov, Nonuniform state of superconductors, Zh.Eksp.Teor.Fiz. 47 (1964) 1136-1146.

[18] D. E. Sheehy, L. Radzihovsky, BEC-BCS crossover in "magnetized" Feshbach-resonantly paired superfluids, Phys. Rev. Lett. 96 (6) (2006) 060401.

[19] D. E. Sheehy, L. Radzihovsky, BEC-BCS crossover, phase transitions and phase separation in polarized resonantly-paired superfluids, Ann. Phys. 322 (8) (2007) 1790-1924.

[20] L. Radzihovsky, D. E. Sheehy, Imbalanced feshbach-resonant fermi gases, Rep. Prog. Phys. 73 (7) (2010) 076501.

[21] G. Sarma, On the influence of a uniform exchange field acting on the spins of the conduction electrons in a superconductor, Journal of Physics and Chemistry of Solids 24 (8) (1963) 1029-1032.

[22] A. Kujawa-Cichy, R. Micnas, Stability of superfluid phases in the 2D spinpolarized attractive Hubbard model, Europhys. Lett. 95 (3) (2011) 37003. 
[23] W. V. Liu, F. Wilczek, Interior Gap Superfluidity, Phys. Rev. Lett. 90 (cond-mat/0208052) (2003) 047002. 5 p.

[24] E. Gubankova, W. V. Liu, F. Wilczek, Breached pairing superfluidity: Possible realization in QCD, Phys. Rev. Lett. 91 (hep-ph/0304016. MIT-CTP3357) (2003) 032001. 4 p.

[25] W. V. Liu, F. Wilczek, P. Zoller, Spin-dependent Hubbard model and a quantum phase transition in cold atoms, Phys. Rev. A 70 (3) (2004) 033603.

[26] E. Gubankova, E. G. Mishchenko, F. Wilczek, Breached Superfluidity via p-Wave Coupling, Phys. Rev. Lett. 94 (1) (2005) 110402.

[27] E. Gubankova, A. Schmitt, F. Wilczek, Stability conditions and Fermi surface topologies in a superconductor, Phys. Rev. B 74 (6) (2006) 64505.

[28] M. Iskin, C. A. R. Sá de Melo, Two-Species Fermion Mixtures with Population Imbalance, Phys. Rev. Lett. 97 (1) (2006) 100404.

[29] M. Iskin, C. A. R. Sá de Melo, Mixtures of ultracold fermions with unequal masses, Phys. Rev. A 76 (1) (2007) 13601.

[30] M. M. Parish, F. M. Marchetti, A. Lamacraft, B. D. Simons, Finitetemperature phase diagram of a polarized Fermi condensate, Nat. Phys. 3 (2) (2007) 124-128.

[31] S. Yin, J. P. Martikainen, P. Törmä, Fulde-Ferrell states and BerezinskiiKosterlitz-Thouless phase transition in two-dimensional imbalanced Fermi gases, Phys. Rev. B 89 (1) (2014) 014507.

${ }_{425}[32]$ A. E. Feiguin, F. Heidrich-Meisner, Pairing states of a polarized Fermi gas trapped in a one-dimensional optical lattice, Phys. Rev. B 76 (22) (2007) 220508.

[33] G. G. Batrouni, M. H. Huntley, V. G. Rousseau, R. T. Scalettar, Exact Numerical Study of Pair Formation with Imbalanced Fermion Populations, Phys. Rev. Lett. 100 (11) (2008) 116405. 
[34] A. Lüscher, R. M. Noack, A. M. Läuchli, Fulde-Ferrell-Larkin-Ovchinnikov state in the one-dimensional attractive Hubbard model and its fingerprint in spatial noise correlations, Phys. Rev. A 78 (1) (2008) 013637.

[35] M. Rizzi, M. Polini, M. A. Cazalilla, M. R. Bakhtiari, M. P. Tosi, R. Fazio, ${ }_{435}$ Fulde-Ferrell-Larkin-Ovchinnikov pairing in one-dimensional optical lattices, Phys. Rev. B 77 (24) (2008) 245105.

[36] M. Tezuka, M. Ueda, Density-Matrix Renormalization Group Study of Trapped Imbalanced Fermi Condensates, Phys. Rev. Lett. 100 (11) (2008) 110403.

${ }_{440}$ [37] A. Kujawa-Cichy, The influence of magnetic field on the superconducting properties and the BCS-BEC crossover in systems with local fermion pairing, arXivarXiv:1309.6680v1

[38] L. He, P. Zhuang, Phase diagram of a cold polarized Fermi gas in two dimensions, Phys. Rev. A 78 (2008) 033613.

445 [39] T. P. Polak, T. K. Kopeć, Frustration effects in rapidly rotating square and triangular optical lattices, Phys. Rev. A 79 (6) (2009) 063629.

[40] T. P. Polak, T. A. Zaleski, Time-of-flight patterns of ultracold bosons in optical lattices in various Abelian artificial magnetic field gauges, Phys. Rev. A 87 (3) (2013) 033614.

450 [41] T. A. Zaleski, T. P. Polak, Synthetic magnetic field effects on neutral bosonic condensates in quasi-three-dimensional anisotropic layered structures, Phys. Rev. A 83 (2) (2011) 23607.

[42] M. M. Maśka, Reentrant superconductivity in a strong applied field within the tight-binding model, Phys. Rev. B 66 (5) (2002) 54533.

455 [43] A. L. Gaunt, T. F. Schmidutz, I. Gotlibovych, R. P. Smith, Z. Hadzibabic, Bose-Einstein condensation of atoms in a uniform potential, Phys. Rev. Lett. 110 (20) (2013) 200406. 
[44] M. Machida, M. Okumura, S. Yamada, Stripe formation in fermionic atoms on a two-dimensional optical lattice inside a box trap: Density-matrix renormalization-group studies for the repulsive Hubbard model with open boundary conditions, Phys. Rev. A 77 (3) (2008) 033619.

[45] T. Esslinger, Fermi-Hubbard Physics with Atoms in an Optical Lattice, Annu. Rev. Condens. Matter Phys. 1 (1) (2010) 129-152.

[46] T. K. Kopeć, T. P. Polak, Ground-state properties of charge and magnetically frustrated two-dimensional quantum Josephson junction arrays, Phys. Rev. B 66 (9) (2002) 094517. 